hep-th/0006047

SU-ITP-00/15

SLAC-PUB-8461

OSU-M-99-7

\title{
Mirror symmetry for open strings
}

\author{
Shamit Kachru ${ }^{1,2}$, Sheldon Katz ${ }^{3}$, \\ Albion Lawrence ${ }^{1,2}$ and John McGreevy ${ }^{1}$ \\ ${ }^{1}$ Department of Physics, Stanford University, Stanford, CA 94305 \\ ${ }^{2}$ SLAC Theory Group, MS 81, PO Box 4349, Stanford, CA 94309 \\ ${ }^{3}$ Department of Mathematics, Oklahoma State University, Stillwater, OK 74078
}

\begin{abstract}
We discuss the generation of superpotentials in $d=4, \mathcal{N}=1$ supersymmetric field theories arising from type IIA D6-branes wrapped on supersymmetric three-cycles of a Calabi-Yau threefold. In general, nontrivial superpotentials arise from sums over disc instantons. We then find several examples of special Lagrangian three-cycles with nontrivial topology which are mirror to obstructed rational curves, conclusively demonstrating the existence of such instanton effects. In addition, we present explicit examples of disc instantons ending on the relevant three-cycles. Finally, we give a preliminary construction of a mirror map for the open string moduli, in a large-radius limit of the type IIA compactification.
\end{abstract}

June 2000 


\section{Introduction}

"The importance of instanton computations in string theory and in $M$-theory can hardly be overstated."[四]

There are many important motivations for studying the physics of D-branes on CalabiYau threefolds in type II string theories (or orientifolds thereof). To begin with, spacefilling branes provide a microscopic construction of brane world models with $\mathcal{N}=1$ supersymmetry. In addition, physical objects in these theories (such as the moduli space of vacua and the superpotential) have a geometric expression. Hence, these theories provide a rich new context for studying quantum geometry via $\mathcal{N}=1$ field theories, along the lines of previous work on $\mathcal{N}=2$ brane probe theories [2]. For a fairly recent introductory review, see [3]; recent work on this subject has appeared e.g. in [4, 5, 6, 6, 7, 8, 9, 19, 10, 11, 12, 13, 14, 15, 16].

Consider a compactification of type IIA string theory on a Calabi-Yau threefold $M$. A single D6 brane wrapped on a supersymmetric three-cycle $\Sigma \subset M$ realizes a $4 \mathrm{~d} \mathcal{N}=1$ quantum field theory. In [6], we began to explore the consequences of mirror symmetry for such brane worldvolume theories (related work appears in [17, 18, 19]). We found that the moduli space of vacua has complex dimension $b_{1}(\Sigma)$, to all orders in $\sigma$-model perturbation theory. Any superpotential must be generated by nonperturbative worldsheet effects, i.e. disc instantons. Now, choose $\Sigma$ so that the mirror cycle $\mathcal{C}$ is a rational curve in the mirror threefold $W$. The mirror of the above D6-brane is a D5-brane wrapped on $\mathcal{C} \times \mathbb{R}^{4}$. When $\mathcal{C}$ has obstructed first-order deformations, the deformation is described by a massless scalar field with a higher-order superpotential; this superpotential is described exactly by classical geometry [4⿴囗十心 6 . Mirror symmetry implies a disc instanton-generated superpotential for the IIA D6 brane. Ideally we could use this to compute the instanton sum exactly. The first obstacle to this program is that the explicit construction of such D-brane mirror pairs is quite difficult, and examples of compact special Lagrangian three-cycles with $b_{1}(\Sigma) \neq 0$ have been scarce.

In this paper, we further this program by providing examples of such pairs, and developing a preliminary understanding of the structure of the instanton sums and the

1 To avoid RR tadpoles, one can take $M$ to be noncompact, or consider a full orientifold model which also has orientifold planes. Alternatively, since we will be working at tree level, one can consider a non-space filling brane whose worldvolume theory still has 4 supercharges, and view the superpotentials we compute in that context. 
mirror map. We begin in $\S 2$ with a more detailed review of supersymmetric D-branes in Calabi-Yau compactifications. These have a standard classification as A-type or B-type branes [20], which roughly correspond to special Lagrangian cycles and holomorphic cycles, respectively. The superpotentials on B-type branes arise from classical geometry [4,6] and we review the geometry of a few specific examples (some with nontrivial superpotentials and some without). We also discuss the qualitative features of superpotentials for A type branes. In $\S 3$, we give an explicit construction of the special Lagrangian three cycles which are mirror to the explicit examples of B-type branes discussed in $\S 2$. In particular, we find examples of smooth three-cycles with nonvanishing $b_{1}$ whose mirrors have moduli space dimension less than $b_{1}$. This effectively proves the existence of disc instanton-generated superpotentials. We also give explicit examples of disc instantons, i.e. holomorphic discs with boundary in a nontrivial homology class on the special Lagrangian cycle. In $\S 4$, we use mirror symmetry to make some statements about the instanton-generated superpotential for our A-type examples. We first discuss a mechanism by which disc instanton effects in our examples could (partially) cancel at special loci in closed string moduli space. We then give a preliminary description of the mirror map for open string moduli in one example. We close with a discussion of promising future directions in $\S 5$.

\section{Superpotentials from D-branes}

\subsection{A-type and B-type branes}

There are two distinct classes of supersymmetric branes in Calabi-Yau compactifications: A-type and B-type branes [20] (which can be constructed as boundary states in the topological A- and B-twisted sigma models respectively, following the notation of [21]). To help distinguish between these cases, we will denote by $M$ a Calabi-Yau used for studying A-type branes, and by $W$ a Calabi-Yau used for studying B-type branes. When we give examples in later sections, mirror pairs will be identified by using a common subscript, $\left(M_{i}, W_{i}\right)$. In geometric language, B-type branes correspond to branes wrapped on holomorphic 0,2,4 and 6-cycles of a Calabi-Yau $W$; while A type branes correspond to branes wrapped on a special Lagrangian three-cycle $\Sigma \subset M$. In both cases, one has to choose 
a gauge field configuration on the D-brane; the supersymmetry-preserving bundles correspond to flat bundles for A-type branes and to stable, holomorphic bundles for B-type branes.2

Assuming the branes to be space-filling, one can prove the following general results about the dependence of the $\mathcal{N}=1$ brane worldvolume action on the Calabi-Yau moduli:

- The superpotential for B-type branes depends only on the complex structure moduli, while FI terms depend only on Kähler moduli.

- The mirror story holds for A-type branes: the superpotential depends only on Kähler moduli, while the FI terms depend on complex structure moduli.

The statements about the superpotential were proven using worldsheet techniques in [4]. The correspondence between FI terms and Calabi-Yau moduli has been explored in [3, 24]; explicit examples of superpotentials for B-type branes were given in [4, 6].

As with closed string $\sigma$-models, the open string $\sigma$-model coupling constants (in which one expands $\sigma$-model perturbation theory) are related to the choice of the Kähler form on $W$, and not to the complex structure. It follows that for B-type branes one can determine the superpotential exactly at $\sigma$-model tree level, using classical geometry. In contrast, for A-type branes (at least for a single brane, which is the case of interest to us here) the superpotential is entirely determined by "stringy" disc instanton corrections [6].

\subsection{Superpotentials for B-type branes}

Information about the superpotential for $B$-type branes is contained in the deformation theory for these branes (and for the gauge bundles on those branes). We will review here the case of branes wrapping curves in a threefold [4], since these are the examples we use in this paper.

For a holomorphic curve $\mathcal{C}$ in a Calabi-Yau threefold $W$, the number of first-order holomorphic deformations is $d=\operatorname{dim} H^{0}\left(\mathcal{C}, \mathcal{N}_{\mathcal{C}}\right)$, where $\mathcal{N}_{\mathcal{C}}$ is the normal bundle of $\mathcal{C} \subset W$. For a single D5-brane wrapping $\mathcal{C}$ in type IIB string theory, this leads to $d$ massless neutral chiral supermultiplets (in addition to the $U(1)$ vector multiplet). A superpotential for chiral multiplets naturally corresponds to an obstruction to extending the associated first-order deformations to higher order.

2 We are being schematic. A more precise discussion of B-type branes as coherent sheaves can be found in [22]; supersymmetric configurations with NS 2-form moduli turned on can be found in 23]. 
Geometrically, the obstruction can only arise if $H^{1}\left(\mathcal{C}, \mathcal{N}_{\mathcal{C}}\right)$ is nontrivial. For simplicity, we only consider a one-parameter deformation. If one chooses a small parameter $\epsilon$, and tries to find a finite holomorphic deformation order by order in $\epsilon$, one computes that the obstruction to finding a solution at each order is represented by an element of $H^{1}\left(\mathcal{C}, \mathcal{N}_{\mathcal{C}}\right)$ [25]. In particular, if there is a nonzero obstruction at order $\epsilon^{n}$, then this geometry is naturally described by a superpotential of the form $W=\Phi^{n+1}$.

The simplest example of such obstructed curves begins with a threefold with $n$ isolated curves, as a particular deformation of the complex structure causes these curves to coincide. They become a single curve $\mathcal{C}$ of multiplicity $n$, and this curve has an obstruction at order $n$ to holomorphic deformations. Physically this is described by $n$ massive vacua coalescing into a single vacuum with superpotential $\Phi^{n+1}$ 目

The B-type examples we will study realize this construction from the following starting point [26,27. Begin with a Calabi-Yau threefold which contains a rational curve $\mathcal{C}$ fibered over a genus-g curve $\mathcal{S}_{g}$. One may canonically associate an element $\omega \in H^{1,0}\left(\mathcal{S}_{g}\right)$ with a non-torid5 first order deformation of the complex structure. The differential $\omega$ generically has $(2 g-2)$ simple zeros, which correspond to the isolated rational curves in the family $\mathcal{S}_{g}$ which survive the deformation. At points of positive codimension in complex moduli, these curves can coincide and form curves of higher multiplicity. 6 There is a natural superpotential for a single D5-brane wrapped on some fiber $\mathcal{C}_{z}$ over a point $z \in \mathcal{S}_{g}$, described in [6]. The local modulus $\phi$ of the rational curve $\mathcal{C}_{z}$ over $z$ can be thought of as an element of the holomorphic tangent space $T_{z}^{1,0} \mathcal{S}_{g}$, and it is the scalar component of a superfield $\Phi$. The superpotential is then:

$$
W(\Phi ; \omega)=\langle\omega, \Phi\rangle+\frac{1}{2 !}\langle\partial\langle\omega, \Phi\rangle, \Phi\rangle+\frac{1}{3 !}\langle\partial\langle\partial\langle\omega, \Phi\rangle, \Phi\rangle, \Phi\rangle+\cdots,
$$

3 Of course the correct normalization of the fields, and thus of the superpotential, depends on the Kähler metric, which we will not compute in this paper.

4 The situation is actually a bit more complicated than this. Our assertion only pertains to the case $\mathcal{N}_{\mathcal{C}}=\mathcal{O} \oplus \mathcal{O}(-2)$. It is an open problem to classify the possible superpotentials that can yield a single curve with multiplicity $n$, even in the next simplest case $\mathcal{N}_{\mathcal{C}}=\mathcal{O}(1) \oplus \mathcal{O}(-3)$. An example in this case is the superpotential $W(\Phi, \Psi)=\Phi^{2} \Psi+\Psi^{3}$, corresponding to a curve with multiplicity 4.

5 We will be studying hypersurfaces in weighted projective space: for these examples the non-toric complex structure deformations are those which are not monomial deformations of the defining equation.

${ }^{6}$ In all of these cases, $\mathcal{N}_{\mathcal{C}}=\mathcal{O} \oplus \mathcal{O}(-2)$, so our previous discussion applies. 
evaluated at $z$. Here $\langle$,$\rangle is the usual inner product between forms and vectors, and \partial$ is the Dolbeault operator on $\mathcal{S}_{g}$. It is easy to see that the expansion in (2.1) can be truncated after $(2 g-1)$ terms without changing the location and multiplicity of the critical points of $W$.

Below are several examples which realize this general framework. These will be our testing ground for a discussion of open-string mirror symmetry. The considerations of [6] yield some predictions for the mirror three-cycles which we will give at the end of these examples: we will describe and explore the mirror examples in $§ 3$.

\section{Ur-Example}

Our examples will all be orbifolds of the Calabi-Yau hypersurface $W$ of degree 8 in $\mathbb{P}_{1,1,2,2,2}^{4}$, defined for example by the equation

$$
p=z_{1}^{8}+z_{2}^{8}+z_{3}^{4}+z_{4}^{4}+z_{5}^{4}=0
$$

$W$ has a singularity at $z_{1}=z_{2}=0$, inherited from the ambient weighted projective space. Blowing up this $Z_{2}$ singularity yields a family of $\mathbb{P}^{1}$ s, parametrized by the genus 3 curve $\mathcal{S}_{3}$ :

$$
z_{3}^{4}+z_{4}^{4}+z_{5}^{4}=0
$$

The non-toric deformations associated to $H^{(1,0)}\left(\mathcal{S}_{3}\right)$ generically lift the family $\mathcal{S}_{3}$ of $\mathbb{P}^{1} \mathrm{~s}$, leaving four isolated $\mathbb{P}^{1} \mathrm{~s}$.

One can see the non-toric deformations explicitly, by considering an equivalent description of $W$ as a complete intersection of a quartic and a quadric in $\mathbb{C P}^{5}$ following [26]. One sees the equivalence by setting the homogeneous coordinates $\left(y_{0}, \cdots, y_{5}\right)$ of $\mathbb{C P}^{5}$ equal to $\left(z_{1}^{2}, z_{2}^{2}, z_{1} z_{2}, z_{3}, z_{4}, z_{5}\right)$. Then the quadric equation

$$
y_{2}^{2}=y_{0} y_{1}
$$

of rank three is automatically satisfied. The model in $\mathrm{P}^{5}$ obviously has complex structure moduli which deform (2.4) to an equation of higher rank. If one deforms the quadric to have rank four or fewer, then one is still describing points in the complex structure moduli space of $W$. Deformations to quadrics of rank greater than four correspond to making an

7 To see this, note that both (2.4) and the rank 4 quadric $y_{0} y_{1}=y_{2} y_{3}$ can be desingularized by the same blowup $y_{0}=y_{2}=0$, hence both blowups fit into the same moduli space. 
extremal transition from $W$ to another Calabi-Yau space. One finds a three-dimensional space of deformations of the quadric which leave one in the moduli space of $W$, hence there are three non-toric deformations. This story is described in full generality (in the case of a family of $\mathbb{P}^{1}$ s parameterized by a genus $g$ curve, and the corresponding $g$ non-toric deformations) in [27].

It is evident that when one deforms (2.4) by a term which increases the rank to four, e.g. $y_{3}^{2}$, one destroys the family of $\mathbb{P}^{1}$ s. This is because the family is located at $y_{0}=y_{1}=y_{2}=0$ (which is the same as $z_{1}=z_{2}=0$ ); the addition of $y_{3}^{2}$ to (2.4) would then force $z_{3}=0$ also. But then the former genus 3 curve 2.3 collapses to the four points $z_{4}^{4}+z_{5}^{4}=0$. Hence, instead of a one-parameter family there are now 4 isolated $\mathbb{P}^{1} \mathrm{~s}$.

Upon wrapping a single D5-brane on a member of the family of $\mathbb{P}^{1}$ s one finds a $U(1)$ gauge theory in four dimensions with a single neutral chiral multiplet $\phi$ parameterizing a local neighborhood in $\mathcal{S}_{3}$. After a generic non-toric deformation described by $\omega$, (2.1) will describe a superpotential with four massive vacua.

In the mirror manifold, $M$, the non-toric deformations have the following description. Toric Kähler moduli in weighted projective space arise from the volume of the space, the blow-up parameters for the fixed loci of the Greene-Plesser orbifold group, and blowup parameters for any singularities of the weighted projective space which intersect the Calabi-Yau. If the CY hypersurface intersects one of these loci $n+1$ times, the toric Kähler deformation changes the size of all $n+1$ divisors simultaneously, while the remaining $n$ "non-toric" moduli change the relative sizes.

In our examples, mirror symmetry demands the following statement about the threecycles $\Sigma \subset M$ mirror to $\mathcal{C} \subset W$. At the locus in Kähler moduli space with the three non-toric moduli turned off, we have a unique first-order deformation which by [28,6] requires $b_{1}(\Sigma) \geq 1$. (If the inequality is not saturated, the instanton sum must give $b_{1}-1$ chiral multiplets a mass.) The instanton-generated superpotential for one of these moduli must vanish until the non-toric deformations are turned on, and then the moduli space generically splits into four massive vacua.

\section{Example I}

The first example which we will discuss is $W_{1}$, the orbifold of $W$ and $\mathcal{S}_{3} \subset W$ by the discrete $Z_{4} \times Z_{2} \times Z_{2}$ group with generators

$$
(1, i, i, i, i), \quad(1,1,-1,-1,1), \quad(1,1,1,-1,-1)
$$


The family of $\mathbb{P}^{1}$ s on $W$ located at $z_{1}=z_{2}=0$ is orbifolded by (2.5), and the curve (2.3) becomes a genus 0 curve after orbifolding. Because $\mathbb{P}^{1}$ has no holomorphic one forms, this model does not admit non-toric deformations which destroy the family of holomorphic spheres. Therefore, there is never a nontrivial superpotential, for any complex structure.

Mirror symmetry requires that $M_{3}$ has a one-parameter family of supersymmetric three-cycles. This would be most simply realized by a family of three-cycles $\Sigma$ with $b_{1}(\Sigma)=1$. No Kähler deformation of $M_{3}$ should lead to a nontrivial disc instanton generated superpotential, so the family of three cycles survives quantum corrections even after deformations of closed string Kähler moduli.

If $M_{1}$ is the mirror of $W_{1}$, we will see in Appendix A that the mirror cycles $\Sigma \subset M_{1}$ to our family of $\mathbb{P}^{1}$ s have $b_{1}(\Sigma)>1$. Therefore we expect disc instanton effects to give masses to $b_{1}(\Sigma)-1$ of the moduli of $\Sigma$ predicted by the classical geometry.

\section{Example II}

Our next example, $W_{2}$, arises from orbifolding $W$ and $\mathcal{S}_{3} \subset W$ by the $Z_{4} \times Z_{4}$ discrete group with generators

$$
(1, i, i, 1,-1), \quad(1,1,1, i,-i) .
$$

Once again we have a family of $\mathbb{P}^{1} \mathrm{~s}$ at $z_{1}=z_{2}=0$. The curve (2.3) becomes a genus-1 curve $\mathcal{S}_{1}$, after orbifolding by (2.6). Thus, if we wrap a D5-brane around a member of this family, there is a single parameter in the superpotential $W$ associated to the holomorphic differential on the curve $\mathcal{S}_{1}$. The corresponding superpotential (2.1) is just

$$
W(\Phi)=c \Phi
$$

where $c$ is related to the magnitude of the non-toric blowup. When $c \neq 0$ there are no supersymmetric vacua: the auxiliary field $F$ in the chiral multiplet is non-vanishing, and since we are coupled to closed string theory the $4 \mathrm{~d}$ gravitino gains a mass. This is in keeping with the fact that after the deformation, the holomorphic spheres have all disappeared.

In the absence of coupling to gravity, one can redefine the supercharges so that the superpotential (2.7) does not break supersymmetry; it simply adds a harmless constant to the Lagrangian. This is reflected clearly in the geometry of the example. In a local neighborhood of the $g=1$ curve of $\mathbb{P}^{1} \mathrm{~S}$ in $W_{2}$, the manifold looks like a product of an $A_{1}$ ALE space and a $T^{2}$. This local geometry is hyperkähler and so has a family of complex structures, parametrized by an $S^{2}$. Upon performing the non-toric deformation (2.7), one 
can choose a different complex structure so that there are still holomorphic curves. Since $W_{2}$ is not hyperkähler, this is prevented by the global geometry at finite volume. Hence, global features of $W_{2}$ are important in determining that supersymmetry is broken, a fact which clearly reflects the need to couple the D-brane worldvolume theory to gravity in order to diagnose the supersymmetry breaking.

If $M_{2}$ is the mirror of $W_{2}$, the mirror cycles $\Sigma \subset M_{2}$ to our family of $\mathbb{P}^{1}$ s should also live in a one-dimensional family, so that $b_{1}(\Sigma) \geq 1$. The non-toric Kähler deformation of $M_{2}$ breaks supersymmetry entirely via disc instanton effects.

\section{Example III}

Example III works much like Example I. Let $M_{3}$ be the orbifold of $W$ by the $Z_{2}$ symmetry generated by $\tilde{g}$ :

$$
\tilde{g}=(1,1,1,-1,-1)
$$

We will consider B-type branes on the mirror $W_{3}$ of $M_{3}$. In $W_{3}$ there is still a one-parameter family of $\mathbb{P}^{1} \mathrm{~s}$, parametrized by a $\mathbb{P}^{1}$ (roughly obtained by orbifolding the genus 3 curve in $W$ ). Again, there is no superpotential for this modulus for any value of the complex structure.

For the mirror A-cycle we will find below that $b_{1}(\Sigma)=1$, so classical results apply for all values of the Kähler moduli of $M_{3}$.

\subsection{Qualitative features of superpotentials for A-type branes}

Coordinates on the moduli space of A-type branes

Let $M$ be a general Calabi-Yau threefold, and $\Sigma \subset M$ a special Lagrangian threecycle. For simplicity, assume $b_{1}(\Sigma)=1$, and assume there is a single holomorphic disc instanton $D$ bounded by a representative $\gamma$ of the generating class in $H_{1}(\Sigma)$. The cycle $\Sigma$ moves in a one-dimensional family in $\sigma$-model perturbation theory; as discussed in [18,6], we can parameterize this family locally by a modulus field $\phi$ :

$$
\phi=\operatorname{Area}(D)+i a
$$

where the area is measured in string units, and $a$ is an axion (the Wilson line of the brane $U(1)$ gauge field around $\gamma)$.

This was the picture given in [6], but a moment's thought indicates that it should be modified. Consider for example a special Lagrangian torus in $T^{6}$. There are clearly 
no holomorphic discs bounding the cycles of $T^{6}$, but this does not mean that there is no moduli space for the special Lagrangian subcycle. Indeed there is a simple ansatz which naturally generalizes the above. Begin with a reference three-cycle $\Sigma_{0}$ in some family $\Sigma_{t}$, defined by an embedding $f_{t}: \Sigma_{0} \hookrightarrow M$. Choose a family of deformations constructed from a harmonic form in some class in $H^{1}\left(\Sigma_{0}\right)$ [28]. Then choose some one-cycle $\gamma_{0} \in \Sigma_{0}$ whose class in $H_{1}\left(\Sigma_{0}\right)$ is dual to this cohomology class via the metric. As $t$ varies in the chosen family of deformations, $f_{t}(\gamma)$ will sweep out some tube $T$ in $M$. A natural coordinate $\phi$ is:

$$
\phi=\int_{T} \omega+i \int_{\gamma_{t}} A_{t}
$$

where $\omega$ is the Kähler form on $M$ and $A_{t}$ is a flat connection on $\Sigma_{t}$. When the tube is holomorphic, the real part is simply the area of the tube.

\section{Finding nontrivial superpotentials}

Before launching into a detailed discussion of specific three-cycles mirror to the above examples, we would like to gain some general and intuitive understanding of the form of the superpotentials directly in the language of the three-cycle geometry.

Following [19], the sum over multiple covers of $D$ yields a superpotential

$$
W= \pm \sum_{n=1}^{\infty} \frac{e^{-n \phi}}{n^{2}}
$$

The sign here depends on details of the fermion determinants around the instanton solution [17]. It follows from 2.11) that

$$
\frac{\partial W}{\partial \phi} \sim \pm \sum_{n=1}^{\infty} \frac{e^{-n \phi}}{n}=\mp \log \left(1-e^{-\phi}\right) .
$$

This has a single critical point at $\phi=\infty$, which from (2.9) is the open string analogue of large radius. Of course one expects that to reach $\phi=\infty, M$ must be at some infinite-radius point.

Now, suppose instead that we have $k$ disc instantons $D_{i}$ bounding the same homology class $\gamma \in H_{1}(\Sigma)$. $D_{i}$ may differ by homology classes in $M$. Choose $\operatorname{Re}(\phi)$ to be the area of $D_{1}$. The exponential of the action for instanton $D_{i}$ is that for $D_{1}$ times a factor $q_{i}$, the exponential of the (complexified) volume of $\left[D_{i}-D_{1}\right] \in H_{2}(M)$. Finally, assume each of these discs are isolated. The resulting superpotential is:

$$
W=\sum_{i=1}^{k} \sum_{n=1}^{\infty} \sigma_{i} \frac{e^{-n \phi}}{n^{2}} q_{i}^{n},
$$


where $\sigma_{i}$ is the sign of the $\sigma$-model fermion determinant for the instanton $D_{i}$. The supersymmetric vacua satisfy:

$$
\sum_{i=1}^{k} \sigma_{i} \log \left(1-q_{i} e^{-\phi}\right)=0 .
$$

This is equivalent to a polynomial equation in $e^{-\phi}$, whose degree is the greater of $\#\{i \mid$ $\left.\sigma_{i}=1\right\}$ and $\#\left\{i \mid \sigma_{i}=-1\right\}$. Note that the critical points need not all be at large radius. The precise locations of the critical points depend on the closed string Kähler moduli through the $q_{i}$.

We get similar results when we include new disc instantons in the class $d \gamma$ for varying $d$. The general result is that if we have $k_{i}$ disc instantons in classes $d_{i} \gamma$, then (assuming for simplicity that all the fermion determinants are positive) there are $\sum k_{i} d_{i}$ supersymmetric vacua. For families of discs some open-string version of the Gromov-Witten invariants of closed string instantons should replace $k_{i}$.

The main point of this discussion is that it is not difficult to imagine one-parameter families of special Lagrangian manifolds which yield, after disc instanton corrections, a discrete set of supersymmetric vacua. This is fortunate as the mirrors of the B-brane configurations discussed in $\S 2.2$ must exhibit this behaviour.

Another lesson is that string instanton effects alter our expectations of the topology of our three-cycles. The natural physical measure of $b_{1}(\Sigma)$ within $\sigma$-model perturbation theory is the number of massless chiral multiplets for a single D6-brane wrapped on this cycle. However, disc instanton effects may well give some of these chiral multiplets a mass, in which case there is no obvious physical distinction between the original three-cycle and a cycle with smaller $b_{1}$.

\section{Special features of $A$-cycles arising as real slices}

We will be focusing on special Lagrangian three-cycles constructed as the fixed point locus of antiholomorphic involutions acting on $M$, in other words antiholomorphic maps $\sigma: z \rightarrow \bar{z}$ which square to the identity. The standard example, which we will use in every case, is the real slice arising as the fixed point set of $z_{i} \rightarrow \bar{z}_{i}$. In this case, for each holomorphic disc we get a conjugate holomorphic disc. If $f: D \rightarrow M$ is a holomorphic map, we can define its conjugate holomorphic disc $g: D \rightarrow M$ by $g(z)=\overline{f(\bar{z})}$. Upon gluing these discs together we find that for any special Lagrangian submanifolds obtained as fixed points of antiholomorphic involutions, holomorphic disc instantons always come as two halves of a rational curve in $M$. Furthermore, it is natural to conjecture that as 
this special Lagrangian cycle moves through a family $\Sigma_{t}$, one may find a set of one-cycles $\gamma_{t} \in \Sigma_{t}$ whose images in $M$ sweep out this rational curve.

The superpotential can be derived from a variant of (2.13). Let $\mathbb{P}$ be the rational curve in question, and $t$ be the integral of the complexified Kähler form of $M$ over $\mathbb{P}$. Let $z$ be the action of the instanton described by $f$; the action for the instanton described by $g$ is then $t-z$. Assuming the fluctuation determinants have the same sign, the superpotential one gets from summing over multiple covers is up to overall sign:

$$
W=\mathrm{Li}_{2}\left(1-e^{-z}\right)+\operatorname{Li}_{2}\left(1-e^{-t+z}\right)
$$

It is easy to see that this has a supersymmetric vacuum at $z=t / 2$. At this point in the open- and closed string moduli space the superpotential is that of the local model in [19].

\section{Constructing the mirror three-cycles}

The next step to fleshing out the mirror map for open strings is, of course, to characterize the mirror map for the submanifolds on which they end. In this section we will find explicit special Lagrangian three-cycles mirror to elements of the families of $\mathbb{P}^{1} \mathrm{~s}$ described in the examples above.

\subsection{Strategy for identifying mirror cycles}

At an arbitrary point in the closed string moduli space, it will be fairly difficult to find explicit mirror cycles. Instead we focus on loci of the moduli space with physical and mathematical significance. In our B-cycle examples, the family of $\mathbb{P}^{1}$ s around which we intend to wrap D5-branes are known to have zero volume at some submanifold in the full (complex and Kähler) moduli space; these points occur when the resolutions of the orbifold singularities discussed above have been turned off (along with the associated NS-NS 2-form moduli). We may identify these points physically by studying BPS D2-branes wrapped on the same cycles in type IIA string theory.8 These D2-branes form massless (vector) multiplets at this discriminant locus as guaranteed by the BPS formula.

In type IIB on the mirror CY, the BPS formula implies that a wrapped D3-brane must become massless at the mirror discriminant locus. Since the mass receives no closed

8 All of our statements are at string tree level so we can be cavalier about changing brane dimension like this. 
string worldsheet instanton corrections, we need simply find the mirror discriminant locus and the vanishing three-cycle via classical geometry. We will discuss the identification of this pair of cycles in $W \subset \mathbb{P}_{1,1,2,2,2}^{4}$ and its mirror; the same logic leads to a similar identification in all of our examples.

The mirror manifold $M$ of $W$ is easily constructed using the Greene-Plesser construction 29]. One quotients $W$ by a suitable maximal group of scaling symmetries, leaving only two complex structure deformations of $M$. These can be represented by the coefficients of the monomials $z_{1} \cdots z_{5}$ and $z_{1}^{4} z_{2}^{4}$ in the defining equation for $M$.

Let us work on the locus in moduli space where the defining equation is:

$$
\left(z_{1}^{4}-z_{2}^{4}\right)^{2}-2 \epsilon z_{1}^{4} z_{2}^{4}+z_{3}^{4}+z_{4}^{4}+z_{5}^{4}=0 .
$$

Here we have set the coefficient of $z_{1} \cdots z_{5}$ to zero; this subspace of the complex structure moduli space of $Y$ intersects the discriminant locus at $\{\epsilon=0, \epsilon=-2\}$. These two points in moduli space can be seen to determine the same $\mathrm{CY}$ manifold by redefining $z_{2}$ by an eighth root of unity. Now, we want to construct a supersymmetric three-cycle which is mirror to a member of the family of $\mathbb{P}^{1} \mathrm{~s}$ on $W$, discussed in $\S 2.2$. At least near large complex structure, $\delta z_{1} \cdots z_{5}$ is the complex deformation of $M$ mirror to the size of the

projective space $W \mathbb{P}_{1,1,2,2,2}^{4}$. Furthermore, we can identify $\epsilon$ as mirror to the modulus controlling the size of the exceptional $\mathbb{P}^{1}$ in $W$, as explained in [26]. Therefore, we are looking for a three-cycle $\Sigma \subset M$ which collapses as $\epsilon \rightarrow 0$. This identification of mirror moduli holds in the other cases as well. We will find a particular such three-cycle $\Sigma$ as a component of the fixed point locus of a real involution acting on the A-model CY. Some set of fixed points of the Greene-Plesser quotient intersect the three-cycle, and the details of the resolution of these singularities will determine the topology of $\Sigma$.

In the following subsections we study the mirrors of the examples considered in $\S 2$. Mirror symmetry reverses the order of increasing complexity, so we will examine the three examples in reverse order.

\subsection{Example III}

In this example the three-cycle topology is the simplest, since the fewest blowups are required. Recall that $M_{3}$ is the orbifold of $W$ by $\tilde{g}=(1,1,1,-1,-1)$. The only fixed points are at $z_{4}=z_{5}=0$, so we introduce a second $\mathbb{C}^{*}$ action and a new coordinate $z_{6}$, where the second $\mathbb{C}^{*}$ acts by $\left(z_{4}, z_{5}, z_{6}\right) \rightarrow\left(\lambda z_{4}, \lambda z_{5}, \lambda^{-2} z_{6}\right)$. The defining equation is modified to:

$$
\left(z_{1}^{4}-z_{2}^{4}\right)^{2}-2 \epsilon z_{1}^{4} z_{2}^{4}+z_{3}^{4}+z_{6}^{2}\left(z_{4}^{4}+z_{5}^{4}\right)=0
$$


so that the manifold is preserved by this second $\mathbb{C}^{*}$. Now consider the real involution:

$$
\sigma:\left(z_{1}, \cdots, z_{6}\right) \rightarrow\left(\bar{z}_{1}, \cdots, \bar{z}_{6}\right)
$$

We obtain a three-cycle $N$ as the fixed point locus of $\sigma$ ( $N$ has two components, which are basically two copies of the desired three-cycle $\Sigma$ ). We will see below that it vanishes as $\epsilon \rightarrow 0$. For the rest of this subsection, we take $z_{1}, \cdots, z_{6}$ to be real (since we wish to work on the fixed point locus of $\sigma$ ).

We use the $\mathbb{C}^{*}$ action of the $\mathbb{P}^{4}$ to set $z_{2}=1$; we will see presently that we will not need to leave this coordinate patch. Furthermore, we define:

$$
x=z_{1}^{4}, \quad Q=z_{3}^{4}+z_{6}^{2}\left(z_{4}^{4}+z_{5}^{4}\right), \quad A=2 \epsilon+\epsilon^{2} .
$$

Solving (3.2) for $x$ in terms of the remaining variables we find:

$$
x=1+\epsilon \pm \sqrt{A-Q} .
$$

We have two branches of solutions for $x$, which meet when $Q=A$. The real slice includes only the region $A \geq Q$, since otherwise $x=z_{1}^{4}$ would be imaginary.

Next, we blow up the orbifold singularity induced by $\tilde{g}$ using the language of symplectic quotients. We introduce a new Kähler parameter $r$ in the following "D-term equation" (c.f. 30 ):

$$
\left|z_{4}\right|^{2}+\left|z_{5}\right|^{2}-2\left|z_{6}\right|^{2}=r
$$

in the description of the full complex manifold $M$ : along the real locus we can dispense with the absolute values. Note that in the full CY we have to gauge away the $U(1)$ under which $\left(z_{4}, z_{5}, z_{6}\right)$ have charges $(1,1,-2)$. Since the $z_{i}$ are real on $N$, the only gauge transformation which acts as an identification on $N$ is $\left(z_{4}, z_{5}, z_{6}\right) \mapsto\left(-z_{4},-z_{5}, z_{6}\right)$, i.e. the orbifold by $\tilde{g}$.

Next, we can solve (3.5) for $z_{4}$ :

$$
z_{4}= \pm \sqrt{r-z_{5}^{2}+2 z_{6}^{2}}
$$

This gives two branches for $z_{4}$ which are glued together along the hyperbola $z_{5}^{2}-2 z_{6}^{2}=r$. Reality of $z_{4}$ requires that $r \geq z_{5}^{2}-2 z_{6}^{2}$. These conditions bound $z_{3}^{2}, z_{5}^{2}, z_{6}^{2}$ from above, allowing us to stay on the patch where $z_{2}=1$.

Consider the regime $0<\epsilon<<1, r>\epsilon$. The region $Q \leq A$ intersects the region of real $z_{4}$ in a region with the topology of a solid cylinder, as pictured below. 


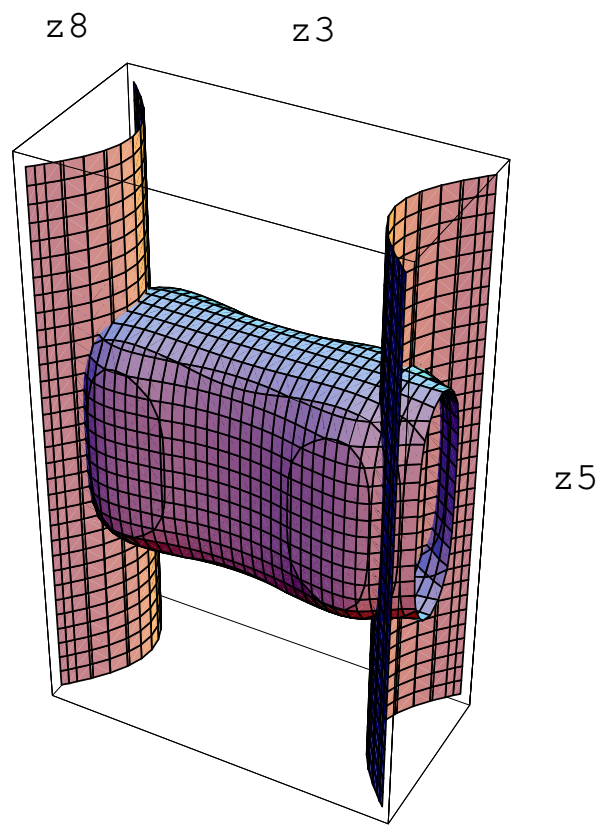

Fig. 1: $z_{4}$ is real between the walls; $x$ is real inside the tube.

Making the orbifold identification merely halves the circumference of this cylinder. The two branches of solutions for $z_{4}$ and the two branches of solutions for $z_{1}^{4}$ give rise to four copies of this cylinder, which are glued along the loci where the $z_{4}$ branches meet and the $z_{1}$ branches meet (the sheet of hyperbolas and $Q=A$ respectively). The gluing along the boundaries of the $z_{4}$ branches yields two solid tori; gluing along the boundaries of the $z_{1}$ branches then yields the closed three-manifold $\Sigma \sim S^{2} \times S^{1}$, which has $b_{1}(\Sigma)=1$. Note that $N$ consists of two copies of $\Sigma$, one with $z_{1}>0$, and the other with $z_{1}<0$ (we will abuse notation and call both copies by the same name, since in any case they are identical).

Because $\Sigma$ is a smooth special Lagrangian three-cycle with $b_{1}=1$, it is guaranteed by McLean's theorem to come in a family of special Lagrangian cycles of real dimension one [28]. Since $Q$ is positive semidefinite on the real slice, when $\epsilon \rightarrow 0$ the locus $Q \leq A$ collapses and the two components of $N$ are no longer finite-volume three-manifolds. We identify the two components of $N$ with two members of the family of $\mathbb{P}^{1} \mathrm{~s}$ in $W_{3}$.

A D6 brane wrapping $\Sigma$ would naively yield, in the transverse $3+1$ dimensions, a $4 \mathrm{~d}$ $\mathcal{N}=1$ field theory with $U(1)$ gauge group and a single neutral chiral multiplet $\phi$. Although $\phi$ has no superpotential to all orders in sigma model perturbation theory (this is the string theory analog of McLean's theorem), $\phi$ can receive a superpotential from disc instantons 
[6]. In this case, we know from the mirror B-model geometry that there are no non-toric deformations which would lift the moduli space of supersymmetric $\mathbb{P}^{1} \mathrm{~s}$. This implies that there is no disc-generated superpotential in this case.

\section{Disc Instantons}

Some explicit examples of disc instantons with boundary on $\Sigma$ can be constructed in this example. Consider the upper half plane parametrized by $u$. Let $z_{1}, \cdots, z_{6}$ be given by

$$
\left(z_{1}, \cdots, z_{6}\right)=\left(a_{1} u, a_{2} u, a_{3} u^{2}, 1,1,0\right) .
$$

We take the $a_{i}$ to be real; this guarantees that the boundary of the disc (where $u$ is real) is mapped to $\Sigma$.

The disc must lie in $M_{3}$, which means that:

$$
\left(a_{1}^{4}-a_{2}^{4}\right)^{2}-2 \epsilon a_{1}^{4} a_{2}^{4}+a_{3}^{4}=0 .
$$

Solutions to (3.8) provide holomorphic maps into $M_{3}$ with boundary on $\Sigma$. In fact this ansatz yields a one-parameter family of discs: the constraint (3.8) eliminates one of the $a_{i}$ and the freedom to rescale the $u$ plane fixes another, but there is one free parameter left in the ansatz. The fact that mirror symmetry implies that there is no disc-generated potential in this case suggests that there is a cancellation between the contributions of different discs. We will discuss such a mechanism in $\S 4$.

\subsection{Example II}

The mirror $M_{2}$ of $W_{2}$ is constructed by orbifolding $W$ by the $Z_{4}$ group generated by $g=(1,1,-1, i, i)$. The group element $g^{2}=(1,1,1,-1,-1)$ is the symmetry by which we orbifolded in Example III, so we should still perform the resolution above. However, $g$ itself fixes the locus $z_{3}=z_{4}=z_{5}=0$, which must be independently blown up. This is achieved by introducing another variable, $z_{7}$, and another $\mathbb{C}^{*}$ action - the charges are summarized in the following table:

$\begin{array}{cccccccc} & z_{1} & z_{2} & z_{3} & z_{4} & z_{5} & z_{6} & z_{7} \\ \mathbb{C}_{1}^{*} & 1 & 1 & 2 & 2 & 2 & 0 & 0 \\ \mathbb{C}_{2}^{*} & 0 & 0 & 0 & 1 & 1 & -2 & 0 \\ \mathbb{C}_{3}^{*} & 0 & 0 & 2 & 1 & 1 & 0 & -4 .\end{array}$


The defining equation is modified to

$$
\left(z_{1}^{4}-z_{2}^{4}\right)^{2}-2 \epsilon z_{1}^{4} z_{2}^{4}+z_{7}^{2} z_{3}^{4}+z_{7} z_{6}^{2}\left(z_{4}^{4}+z_{5}^{4}\right)=0
$$

We will use $z_{3}, z_{5}$, and $z_{6}$ as coordinates on the real slice, $N$, which is the fixed point locus of

$$
\sigma:\left(z_{1}, \cdots, z_{7}\right) \rightarrow\left(\bar{z}_{1}, \cdots, \bar{z}_{7}\right)
$$

Redefining

$$
x=z_{1}^{4}, \quad Q=z_{7}^{2} z_{3}^{4}+z_{7} z_{6}^{2}\left(z_{4}^{4}+z_{5}^{4}\right), \quad A=2 \epsilon+\epsilon^{2}
$$

we find:

$$
x=z_{2}^{4}(1+\epsilon \pm \sqrt{A-Q}) .
$$

On the real slice, the D-term equations for $\mathbb{C}_{2,3}^{*}$ read

$$
z_{4}^{2}+z_{5}^{2}-2 z_{6}^{2}-r_{2}=0,2 z_{3}^{2}+z_{4}^{2}+z_{5}^{2}-4 z_{7}^{2}-r_{3}=0
$$

Solving (3.12) for $z_{4}$ and $z_{7}$ we find:

$$
z_{4}= \pm \sqrt{r_{2}-z_{5}^{2}+2 z_{6}^{2}}
$$

and

$$
z_{7}= \pm \sqrt{\frac{1}{2}\left(r_{2}-r_{3}+z_{3}^{2}+z_{6}^{2}\right)} .
$$

If we choose Kähler moduli so that $r_{2}>r_{3}$, then $z_{7}$ never vanishes, and the two branches of solutions never meet. On the branch where $z_{7}>0, Q=z_{7}^{2} z_{3}^{4}+z_{7} z_{6}^{2}\left(z_{4}^{4}+z_{5}^{4}\right)$ is positive semidefinite as in $\S 3.2$, and (3.11) tells us that this component of the real slice vanishes as $\epsilon \rightarrow 0$. The other component of the real slice does not shrink on this locus and so is of no interest to us. Under the restriction that $z_{4}= \pm \sqrt{r_{2}-z_{5}^{2}+2 z_{6}^{2}}$ is real, the three-cycle of interest again resides on the patch where $z_{2}=1$ (for this regime in closed string moduli space). The determination of topology goes through in complete analogy with Example III, and we again find two components of the real slice $N$, each of which is topologically $S^{2} \times S^{1}$. We again call the components $\Sigma$.

So we see again that a D6-brane on $\Sigma$ has a one-dimensional moduli space to all orders in $\sigma$-model perturbation theory. The non-toric deformation of $W_{2}$ which lifts the moduli space of supersymmetric $\mathbb{P}^{1} \mathrm{~s}$ must in fact map to a small deformation of the Kähler structure of $M_{2}$. This deformation cannot change the topology of $\Sigma$, since $\Sigma$ is 
a smooth three-cycle and the deformation can be made arbitrarily small. Hence, for the moduli spaces of the mirror pair to match, the non-toric Kähler deformation must activate a disc-generated superpotential. We give further evidence for this below.

\section{Disc Instantons}

For this example we can again construct explicit examples of disc instantons with boundary on $\Sigma$. Using the holomorphic quotient description of $M_{2}$, we fix the three $\mathbb{C}^{*}$ actions to set $z_{4}=z_{5}=z_{7}=1$.

Consider the upper half plane parametrized by $u$. Let $z_{1}, \cdots, z_{7}$ be given by

$$
\left(z_{1}, \cdots, z_{7}\right)=\left(a_{1} u, a_{2} u, a_{3} u^{2}, 1,1,0,1\right)
$$

Again the $a_{i}$ are real, so that the boundary of the disc $u \in \mathbb{R}$ is mapped to $\Sigma$.

In order that the disc lies in $M_{2}, a_{1,2,3}$ must again satisfy Eq. (3.8). As before, we find a one-parameter family of holomorphic maps of the disc into $M_{2}$ with boundary on $\Sigma$.

\subsection{Example I}

The mirror $M_{1}$ of $W_{1}$ is constructed by orbifolding $W$ by the $Z_{2} \times Z_{2}$ group generated by $g_{1}=(1,1,-1,-1,1)$ and $g_{2}=(1,1,1,-1,-1)$. This example provides the richest spectrum of phenomena for the A-cycles in $M_{1}$ as we have to perform the most blowups. Augmenting the weighted projective space by the following additional variables and $\mathbb{C}^{*}$ actions allows us to resolve all singularities which intersect the three-cycle: 


$\begin{array}{lcccccccc} & z_{1} & z_{2} & z_{3} & z_{4} & z_{5} & z_{6} & z_{7} & z_{8} \\ \mathbb{C}_{1}^{*} & 1 & 1 & 2 & 2 & 2 & 0 & 0 & 0 \\ \mathbb{C}_{2}^{*} & 0 & 0 & 0 & 1 & 1 & -2 & 0 & 0 \\ \mathbb{C}_{3}^{*} & 0 & 0 & 1 & 0 & 1 & 0 & -2 & 0 \\ \mathbb{C}_{4}^{*} & 0 & 0 & 1 & 1 & 0 & 0 & 0 & -2 .\end{array}$

The defining equation for $M_{1}$ is:

$$
\begin{array}{r}
0=\left(z_{1}^{4}-z_{2}^{4}\right)^{2}-2 \epsilon z_{1}^{4} z_{2}^{4}+z_{7}^{2} z_{8}^{2} z_{3}^{4}+z_{6}^{2} z_{8}^{2} z_{4}^{4}+z_{6}^{2} z_{7}^{2} z_{5}^{4} \\
\equiv\left(z_{1}^{4}-z_{2}^{4}\right)^{2}-2 \epsilon z_{1}^{4} z_{2}^{4}+Q .
\end{array}
$$

We will use $z_{3}, z_{4}$ and $z_{5}$ as our independent variables. Solving the D-term equations associated to $\mathbb{C}_{2}^{*}, \mathbb{C}_{3}^{*}$ and $\mathbb{C}_{4}^{*}$ for $z_{6}, z_{7}$ and $z_{8}$ on the real slice gives

$$
\begin{aligned}
z_{6} & = \pm \sqrt{\frac{1}{2}\left(-r_{2}+z_{4}^{2}+z_{5}^{2}\right)} \\
z_{7} & = \pm \sqrt{\frac{1}{2}\left(-r_{3}+z_{3}^{2}+z_{5}^{2}\right)} \\
z_{8} & = \pm \sqrt{\frac{1}{2}\left(-r_{4}+z_{3}^{2}+z_{4}^{2}\right) .}
\end{aligned}
$$

where $r_{2,3,4}$ are the Kähler parameters controlling the sizes of the associated exceptional divisors in $M_{1}$.

Choosing $r_{2,3,4}>0$ we find a geometric phase. The real slice will have several identical components of which we choose one and call it $\Sigma$. Since the function $Q$ defined above is positive semidefinite, this component will shrink when $\epsilon \rightarrow 0$.

The analysis of the topology of this component $\Sigma$ of the real slice is included in the appendix. The conclusion is that in the regime of Kähler moduli considered above, $b_{1}(\Sigma)=5$. In particular, $\Sigma$ is a connected sum of 5 copies of $S^{1} \times S^{2}$. The mirror $\mathbb{P}^{1}$ has a one-dimensional moduli space, while by McLean's theorem $\Sigma$ would move in a 5dimensional family. Thus we are guaranteed the presence of a disc-instanton generated superpotential which lifts four of the flat directions.

\section{Mirror symmetry and the superpotential}

We are interested in computing the superpotential for the A-type examples in $\S 3$, by finding a mirror map for the open string moduli. In this section we will make some progress in this direction. We will start in $\S 4.1$ by arguing that the features of the mirror 
B-type examples near the toric locus are captured by certain features of disc instantons in our A-type examples. In $\S 4.2$ we will find a large-complex-structure limit of the B-type examples for which the disc instantons of the mirror three-cycle will be large, and construct a mirror map for the chiral multiplet in this limit.

\subsection{The superpotential near the toric locus}

Recall that for the Ur-example and for example II, mirror symmetry requires the following story. The ambient Calabi-Yau $M$ (resp. $M_{2}$ ) has $g$ non-toric deformations with $g=3$ (resp. 1); these arise because the hypersurface intersects $g$ of the divisors of the (orbifolded) weighted projective space twice, to create two divisors in the hypersurface. At the "toric locus" these divisors have the same size. At this point the three-cycles we study must have a one-dimensional moduli space (namely a genus-g curve). As we leave this locus, we acquire a superpotential and are left with $2 g-2$ isolated three-cycles. We argue here that there will be different disc contibutions which cancel on the toric locus.

In our A-cycle examples, the defining equation for the threefold $M$ may be written as:

$$
0=\left(z_{1}^{4}-z_{2}^{4}\right)^{2}-2 \epsilon z_{1}^{4} z_{2}^{4}+Q
$$

where $Q$ is a function of all the variables $z_{k>2}$ other than $z_{1}$ and $z_{2}$. It follows that on the hypersurface:

$$
\left(z_{1}^{4}\right)_{ \pm}=z_{2}^{4}(1+\epsilon \pm \sqrt{A-Q})
$$

with $A=2 \epsilon+\epsilon^{2}$. Consider the map, $i: M \rightarrow M$, which fixes $z_{k>2}$ but flips the branches of $z_{1}^{4}$. We claim that this is an isometry of $M$ at the toric locus. Note that $i$ induces a map on the toric part of the cohomology: $i^{*}: H_{\text {toric }}^{2}(M) \rightarrow H_{\text {toric }}^{2}(M)$. This happens to be the identity and so preserves the Kähler class on $M$. Furthermore, it preserves the complex structure, and so by Yau's theorem [31] it preserves the metric on $M$.

The non-toric Kähler deformations are odd under $i^{*}$. To see this, let us describe them in more detail following [27]. In our examples $M$ is a hypersurface in the quotient $\mathbb{P}_{1,1,2,2,2}^{4} / \Gamma$, where $\Gamma$ is the relevant Greene-Plesser (GP) (sub)group. If $\Gamma$ has elements which fix the locus $z_{3}=z_{4}=z_{5}=0$ (and not just varieties which contain it such as $z_{3}=z_{4}=0$ ), this locus must be blown up to desingularize $M$. The Kähler parameters controlling these blow-ups have a natural mirror description in toric geometry [26, 27]. Within the lattice of exponents of monomials in the ambient space of $W$, the allowed monomials (preserved by the subgroup of the GP group complementary to $\Gamma$ ) lie on a 
polyhedron. The lattice points on the lines and faces of this polyhedron correspond to divisors in $M$ and monomial deformations of $W$. In particular the lattice points corresponding to $z_{3,4,5}^{4}$ in $W$ form the vertices of a triangular face of this polyhedron and control the monomial deformations of the Riemann surface $\mathcal{S}_{g} \subset W$. The $g$ interior points can be used to construct the holomorphic differentials of $\mathcal{S}_{g}$, so they are associated to the non-toric deformations which destroy the family of $\mathbb{P}^{1}$ s over $\mathcal{S}_{g}$.

In $M$ these $g$ interior points denote the Kähler parameters controlling the blowup of $z_{3}=z_{4}=z_{5}=0$. The exceptional divisors intersect the hypersurface twice in $M$, at the loci $\left(z_{1}^{4}\right)_{ \pm}=z_{2}^{4}(1+\epsilon \pm \sqrt{A})$. The non-toric moduli control the relative sizes of these divisors in $M$. Since the map $i$ defined above interchanges these two loci, and the non-toric deformations change their relative sizes, $i$ can no longer be an isometry away from the toric locus. Furthermore, at the toric locus $i$ will change the sign of the non-toric deformation.

The map $i$ lifts naturally to a map on holomorphic discs. Hence, these discs should come in pairs related by $i$. It is plausible that on the toric locus, the sign of the contribution in Eq. (2.13) is changed by $i$; then the disc instanton contributions of the pair will cancel in the superpotential. This could come about through the action of $i$ on fermion zero modes or on the pfaffian. When $i$ is not an isometry, the areas of the discs related by $i$ will differ, so their contributions to $W$ will no longer cancel.

Note that this cancellation cannot happen for every disc at the toric locus. In particular, in example I we find that $b_{1}(\Sigma)=5$ while the true moduli space is one dimensional. 8 This suggests that the involution $i$ changes the sign of the contribution of discs with boundaries in only one class of $H_{1}(\Sigma)$. There is clearly much to understand here.

The set of examples we have considered fits into the more general framework discussed in [27]. The B-model CY in general contains a family of $A_{N}$ singularities fibered over a genus $g$ curve. Upon resolving this family, one then finds $N$ families of $\mathbb{P}^{1}$ s. There are $g$ interior points on the relevant face of the toric diagram, and therefore $g$ non-toric complex structure deformations which destroy each family. Furthermore, the defining equation for the A-model CY will be of degree $N+1$ in one of the variables, $x$, which is single-valued under the GP orbifold (the analog of $x=z_{1}^{4}$ ), leading to $N+1$ branches of solutions. $g$ toric divisors each intersect these $N+1$ branches once. On the toric locus, the Galois group, $S_{N+1}$, of the defining equation will act via isometries on the CY by interchanging the branches of solutions for $x$, leading to cancellations between discs. Upon turning on the non-toric Kähler moduli, these isometries are broken allowing a nontrivial superpotential.

9 At special degenerate points in the complex structure moduli space of $W$, the dimension of the moduli space of the mirror $\mathbb{P}^{1}$ enlarges to two, but never to five. 


\subsection{The mirror map}

One of our long-term goals is to use mirror symmetry to find the explicit form of the instanton sums for A-type branes. Of course this sum is automatically computed in the B-model, but we require a mirror map for the open string fields in order that this be of any use. In the context of our models, this means the following. The B-brane moduli space is parametrized by the complex coordinate $z$ on a genus $g$ surface, while the A-brane moduli space is parametrized via (2.9) by the disc area $A$ and Wilson line $a$. Thus, defining

$$
q=e^{-\phi}
$$

we would like to find a map $z(q)$.

As in the closed string case, this will be easiest around "large radius" or "large complex structure" points, in particular when $\operatorname{Re}(\phi)$ is large so that the instanton action is small and classical geometry is a reasonable guide. We therefore search for a map in a region of large radius of our IIA models, and in the mirror large complex structure limit of our IIB models. In this limit we can identify the mirror of the large-disc limit of the A-cycle moduli space with a particular point on the B-cycle moduli space. Finally, since the superpotential is explicitly computable in the B-model side, we can use our previous intuition about the A-type superpotentials to guess at an explicit mirror map in this limit.

We will work exclusively with the Ur-Example in this section.

\section{The large-complex-structure limit of $W$}

On the manifold $M$, we are interested in the large radius limit. In particular, the sizes of discs ending on the real slice are determined by sizes of rational curves (as shown in $\S 2.3)$, so we demand that the exceptional divisors which intersect the three-cycle are large. The mirror locus in the complex structure moduli space of $W$ is specified by the defining equation

$$
p=\alpha z_{3}^{2} z_{4} z_{5}+\beta z_{3} z_{4}^{2} z_{5}+\gamma z_{3} z_{4} z_{5}^{2}=0
$$

with $\alpha, \beta, \gamma$ large and at fixed ratios (recall that these monomials correspond to the toric divisors in $\left.M_{1}\right)$. (4.4) can be rewritten as

$$
z_{3} z_{4} z_{5}\left(\alpha z_{3}+\beta z_{4}+\gamma z_{5}\right)=0
$$

This degeneration is similar to the large complex structure limit discussed in [32]. It is described by four $\mathbb{P}^{3}$ s at $z_{3}=0, z_{4}=0, z_{5}=0$ and $\alpha z_{3}+\beta z_{4}+\gamma z_{5}=0$ which intersect as shown in the figure. 


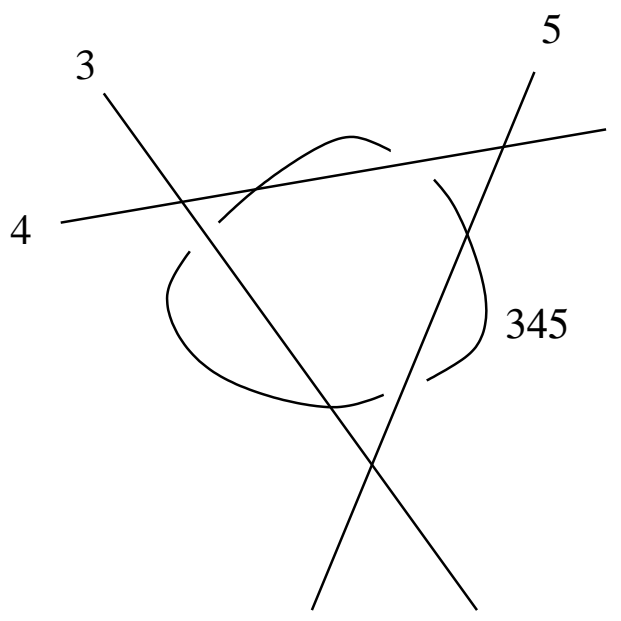

Fig. 2: $M$ in the large complex structure limit. The lines represent $\mathbb{P}^{3}$ s labeled by which coordinate vanishes on them. '345' denotes the one where $\alpha z_{3}+\beta z_{4}+\gamma z_{5}=$ 0 . This picture also accurately portrays the degeneration of the curve $\mathcal{S}_{3}$ where $z_{1}=z_{2}=0$, in which case the lines represent $\mathbb{P}^{1} \mathrm{~s}$.

Each $\mathbb{P}^{3}$ is identical to the others and joins them in a symmetrical way. As in [32] where the example of the quintic at large complex structure is discussed, in this large complex structure limit of $W$ it is easy to write down a flat Kähler metric. Let us examine the metric on $z_{3}=0$ near the locus $z_{4}=0$. We may use the $\mathbb{C}^{*}$ action on the $W \mathbb{P}^{4}$ to set $z_{5}=1$. The standard residue formula yields the following expression for the holomorphic $(3,0)$ form $\Omega$ in this degenerate limit:

$$
\Omega=\int_{p=0} \frac{d z_{1} \ldots d z_{5}}{p}=\frac{d z_{1} d z_{2} d z_{4}}{z_{4}\left(\beta z_{4}+\gamma\right)}
$$

Near $z_{4}=0$ the Ricci-flat metric is clearly:

$$
d z_{1}^{2}+d z_{2}^{2}+\frac{1}{\gamma^{2}} d\left(\ln z_{4}\right)^{2}
$$

Elsewhere on the slice it is:

$$
d z_{1}^{2}+d z_{2}^{2}+d \zeta^{2}
$$

where

$$
\zeta=\frac{1}{\gamma} \ln \frac{\beta z_{4}+\gamma}{z_{4}}
$$

$\mathcal{S}_{g}$ in this limit lives at $z_{1}=z_{2}=0$. It is a chain of four $\mathbb{P}^{1} \mathrm{~s}$, one in each $\mathbb{P}^{3}$ and joined as in fig. 2. The genus-3 structure is clear from this figure. The metric is simply induced from Eq. (4.9). In particular it is clear that the different components are joined 
along infinite cylinders, parameterized by $\ln z_{i}$ for $z_{i} \rightarrow 0$. This then is the asymptotic moduli space for a D5-brane wrapped on an element of the family of $\mathbb{P}^{1}$ s at $z_{3}=0$ near $z_{4}=0$.

\section{The large-radius limit of $M$}

In the mirror $M$ there are three toric divisors which are taken to be large: near the toric locus, this means that all relevant divisors are large since the toric modulus controls the sum of the sizes of the exceptional divisors.

The moduli of the A-type branes are the areas of discs; according to our discussion at the end of $\S 2.3$ these discs live in pairs forming $\mathbb{P}^{1} \mathrm{~s}$ in the Poincaré dual class of these toric divisors. When the brane wraps a real slice it bisects these $\mathbb{P}^{1}$ s. Let us parameterize the $\mathbb{P}^{1}$ by an altitudinal angle $\theta$ and an azimuthal angle $\rho$. The equator is $\theta=0$ and the real slice intersects this equator. Let $2 \pi R$ be the circumference of the equator. $R$ will be a function of $\alpha, \beta, \gamma$ via the mirror map for closed strings. As the three-cycle moves through its moduli space, it will sweep out the $\mathbb{P}^{1}$ by intersecting it at fixed $\theta$. We choose the open-string modulus so that $\phi$ is the area of the smaller disc. For $\theta$ close to zero, a natural metric on the disc coordinate is:

$$
d s^{2}=d A^{2}+d a^{2}=2 \pi R^{4} \cos \theta(d \theta)^{2}+\frac{1}{(2 \pi R)^{2} \cos ^{2} \theta}(d \alpha)^{2}
$$

where $\alpha \in[0,2 \pi]$. Near $\theta=0$ we can set $\cos \theta=1$ and this is clearly a cylinder, with the periodic direction given by the Wilson line.

\section{Asymptotic identification of the coordinates}

Let us take $\beta, \gamma \in \mathbb{R}$. Up to overall normalizations of the fields we roughly identify

$$
\ln z_{4}=\phi
$$

due to the periodicity of the imaginary parts of each side of this equation. This use of the periodicity is similar to the use of monodromy properties in identifying the closed string mirror map. Note that the correct normalization of the fields is extracted from the so far unknown Kähler metric. Thus our map is only good up to some overall constant. 


\section{Computing the superpotential}

As reviewed in $\S 2$, the deformation along the non-toric locus can be specified by the choice of a holomorphic differential. Let $\tilde{p}$ be the defining polynomial for $\mathcal{S}_{g} \subset W$ at $z_{1}=z_{2}=0$. The general holomorphic differential can be written as:

$$
\omega=\int_{\tilde{p}=0} \frac{z_{3} d z_{4} d z_{5}+z_{4} d z_{3} d z_{5}+z_{5} d z_{3} d z_{4}}{\tilde{p}}\left(a z_{3}+b z_{4}+c z_{5}\right)
$$

On the locus $z_{3}=0$, this becomes:

$$
\omega=\frac{d z_{5}}{z_{5}\left(\beta z_{4}+\gamma z_{5}\right)}\left(b z_{4}+c z_{5}\right)+\frac{d z_{4}}{z_{4}\left(\beta z_{4}+\gamma z_{5}\right)}\left(b z_{4}+c z_{5}\right)
$$

We can concentrate on the region near $z_{4}=0$ by using the $\mathbb{C}^{*}$ action of $W$ to fix $z_{5}=1$. Then

$$
\omega=\frac{d z_{4}}{z_{4}\left(\beta z_{4}+\gamma\right)}\left(b z_{4}+c\right)
$$

In the flat coordinates $x$ we write $\omega=f(x) d x$. As shown in [6], $W^{\prime}(\Phi)=f(\Phi)$ for the associated chiral multiplet. This superpotential clearly has a single vacuum at $z_{4}=-c / b$. It is easy to see that there is a single such vacuum in each of the $\mathbb{P}^{1}$ components of $\mathcal{S}_{3}$ in this limit, for a total of $2 g-2=4$ isolated vacua.

We wish to make contact with the large-disc limit of the toric locus of the A-cycle moduli space. Therefore we push the vacuum to infinite distance by sending $c \rightarrow 0$. In this limit,

$$
W_{\zeta}(\zeta)=b z(\zeta)=\frac{\gamma}{e^{\gamma \zeta}-\beta}
$$

for the superpotential of the B-brane. If the A-cycle superpotential were dominated by a single pair of discs, the corresponding superpotential would be that in Eq. (2.15). Certainly as $z_{4} \rightarrow 0$ and $\phi \rightarrow \infty,(4.15)$ and (2.15) are equal to lowest order in $z_{4}$ and $e^{-\phi}$, given (4.11).

Our candidate superpotentials are equal only to lowest order as we only have an asymptotic mirror map at present. There are several complications in constructing an exact mirror map. First, given our experience with Example I, we expect that the three-

cycle has a sizable number of classical moduli. All but one gain masses from instanton corrections, but the remaining moduli space may be a nontrivial submanifold of the classical moduli space. So our formulae for the superpotential as a function of this modulus are undoubtedly rather schematic. 
Secondly, we have assumed that the only contributions are a disc $D \in H_{2}(M, \Sigma)$ plus its three images arising from the anti-holomorphic involution and the map $i$ discussed in §4.1. Of course there may be higher-degree contributions $n D$ which are not multiple covers, and there may be contributions from discs $D^{\prime}$ for which $\left[D-D^{\prime}\right]$ lies in a nontrivial class in $H_{2}(M)$. We leave these issues for future work.

Finally, we have not yet found a global topology of the moduli space of A-branes which matches the topology of the moduli space of the B-branes, even in this degenerate limit.

\section{Discussion}

In this paper, we have presented explicit examples of three-cycles with nontrivial topology which are mirror to two-cycles which have either obstructed holomorphic deformations or no deformations. After the results of [6], this clearly shows that there is a disc instanton generated superpotential for the moduli of such cycles. We have certainly not given a complete formulation of the mirror map in these examples, but we have made a first step by presenting an analog of the monomial-divisor mirror map for closed string moduli [33].

Although we lack the full power of $\mathcal{N}=2$ special geometry that exists for closed string mirror symmetry, the structure of the $\mathcal{N}=1$ theory we are studying gives us some information. In particular, the superpotential $W$ must be holomorphic in the appropriate variables. Therefore, in limits where one has an open string modulus $\phi$ which is periodic with period $2 \pi i$, the superpotential must be a holomorphic function of $e^{\phi}$. This together with some detailed knowledge of the behavior of $W$ at singularities should be enough to determine the function entirely.

We can also draw some general lessons from this work and the results of [6]. As with closed strings, in making mathematical statements using mirror symmetry one must take the instanton corrections into account. For instance, there is a general conjecture that fundamentally, mirror symmetry is a relation between the Lagrangian submanifolds of a threefold and the semistable coherent sheaves on its mirror [34]. The fact that stringy nonperturbative effects prevent generic special Lagrangian three-cycles from being supersymmetric indicates that this comparison will be complicated. 
It would be interesting to study these issues in the presence of orientifolds (required for tadpole cancellation), as a step towards genuine model-building. 10 Many of the results of this paper and [4,6] rest on the fact that from the $\sigma$-model point of view, the superpotential is essentially a topological quantity and can be computed in an appropriately twisted theory. Since $\mathcal{N}=2$ worldsheet supersymmetry is a consequence of $\mathcal{N}=1, d=4$ spacetime supersymmetry [35], the twisted theories will still make sense in the presence of orientifolds.

Another subject worth exploring is the behavior of the topology of a given special Lagrangian cycle $\Sigma$ as the closed string parameters vary. It is clear from some of our examples that the topology of $\Sigma$ can change as one varies Kähler parameters of the ambient Calabi-Yau space. For instance, in the example we discuss in Appendix A, different choices of the blow-up parameters $r_{2,3,4}$ yield three-cycles of different topology in the same homology class.

\section{Acknowledgements}

We would like to thank M.R. Douglas, Y. Eliashberg, A. Klemm, D. Morrison, J.R. Myers, M.R. Plesser, S. Sethi, C. Vafa, E. Zaslow, and Y. Zunger for helpful communications. We thank P. Kaste for pointing out an error in an earlier version of this paper. S. Kachru was supported in part by an A.P. Sloan Foundation Fellowship, and by the DOE under contract DE-AC03-76SF00515. S. Katz was supported in part by NSA grant number MDA904-98-1-0009, and would like to thank the Stanford High Energy Theory Group for their hospitality during the course of this project. A. Lawrence was supported in part by the DOE under contract DE-AC03-76SF00515 and in part by a DOE OJI grant awarded to E. Silverstein; he would like to thank the University of Chicago High Energy Theory Group, the Rutgers High Energy Theory Group, and especially the Oklahoma State University Department of Mathematics for their hospitality during the course of this project. J. McGreevy was supported in part by the Department of Defense NDSEG Fellowship program. This project received additional support from the American Institute of Mathematics.

10 We would like to thank S. Sethi for a discussion of these points. 


\section{Appendix A. Some Details Concerning Example I}

In this appendix we determine the topology of $\Sigma$, the component of the real slice of the mirror of $\mathbb{P}_{1,1,2,2,2}[8]$ on which all of the $w_{i}$ are positive. We will choose a regime in moduli space where

$$
\epsilon>>r_{i}, \quad r_{1}>>r_{i}
$$

for all $i \geq 2$.

As in the simpler examples, we solve the defining equation for $x=z_{1}^{4}$ by

$$
x=1+\epsilon \pm \sqrt{A-Q} .
$$

We have set $z_{2}=1$ again. We will see that the other variables charged under $\mathbb{C}_{1}^{*}$ are bounded on $\Sigma$ and so $\Sigma$ is entirely contained in this coordinate patch. The locus $B \equiv$ $\{Q=A\}$ where the two branches of $x$ are joined is a big (not quite round) ball in the $\mathbb{R}^{3}$ coordinatized by $z_{3,4,5}$. The branches of $x$ are then two copies of this ball glued along the boundary. The loci where the branches of $z_{6,7,8}$ are joined are three tubes surrounding the coordinate axes and ending on $B$. The region where all variables are real is the part of the inside of $B$ which is outside the union of these tubes. Suppose we are in a regime of moduli where $r_{3}+r_{4}<r_{2}$. Then the tubes surrounding the $z_{3}$ and $z_{4}$ axes will both intersect the one surrounding the $z_{5}$ axis, but not each other, like this:

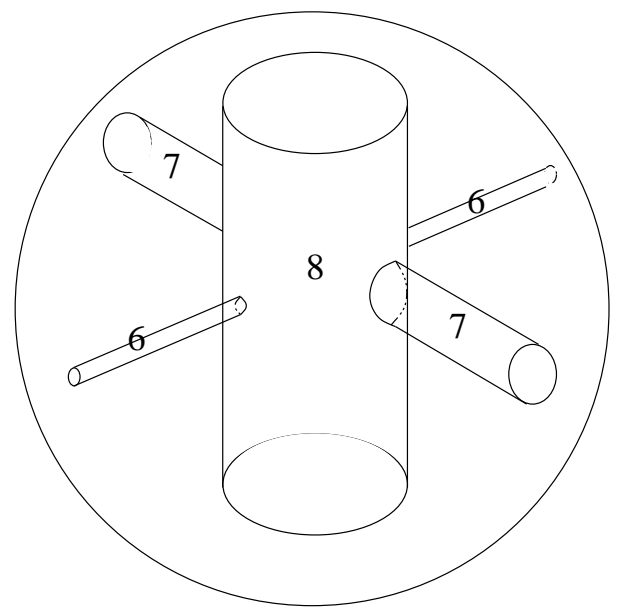

Fig. 3: The real slice is the ball with the tubes removed. The tubes are labeled according to which branches are glued along them.

Now divide by the orbifold group which maps the real slice to itself. It acts by flipping signs in pairs:

$$
\left(z_{3}, z_{4}, z_{5}\right) \sim\left(z_{3},-z_{4},-z_{5}\right) \sim\left(-z_{3}, z_{4},-z_{5}\right) \sim\left(-z_{3},-z_{4}, z_{5}\right) .
$$




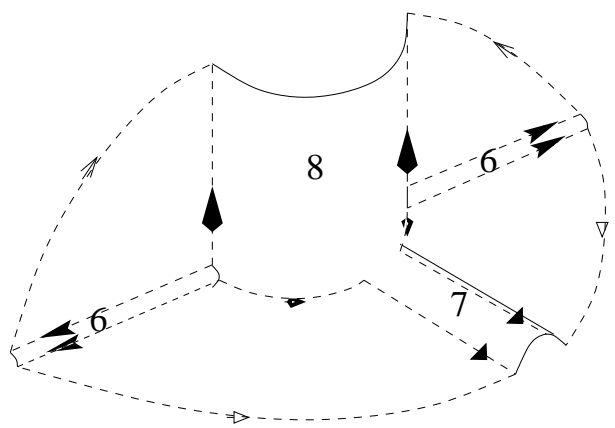

Fig. 4: A fundamental domain for the orbifold action on the real slice (glue along the dotted lines with matching arrows).

After performing this identification, the plumbing fixture surrounding the origin depicted in fig. 3 becomes a half-cigar (where $z_{8}=0$ ) ending on $B$ with two smaller tubes coming off of it (where $z_{6}=0$ and $z_{7}=0$ respectively) and ending on $B$ as well.

Next, glue the two $x$-branches along $B$. This produces an $S^{3}$ with the following set removed: The locus where $z_{8}$ becomes imaginary is now a full cigar, and the $z_{6}=0$ and $z_{7}=0$ loci are two handles coming off of this cigar.

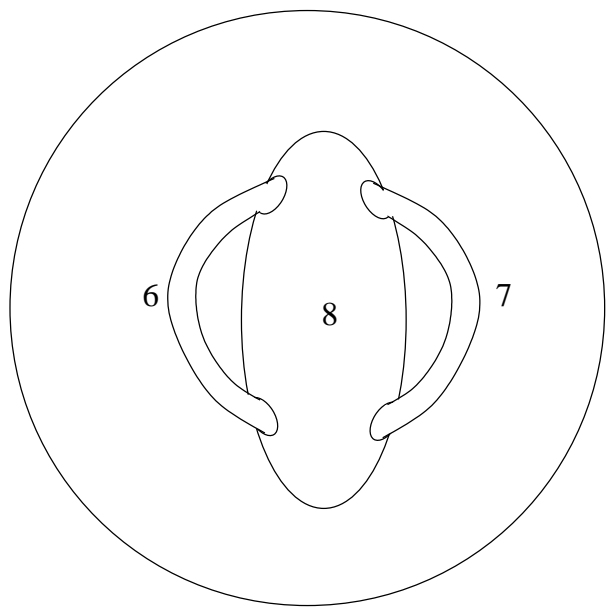

Fig. 5: The real slice is the ball with the blob in the middle excised.

An $S^{3}$ with the $z_{8}$-cigar removed is again a three-ball; the two handles coming off of the cigar become tunnels through this three-ball.

We take four copies of this creature to represent the two branches each of $z_{6}$ and $z_{7}$. They are glued in pairs along the tunnels. To see what this is we must use the fact that gluing handlebodies along a tunnel is the same as gluing along a tube that contains a handle. 


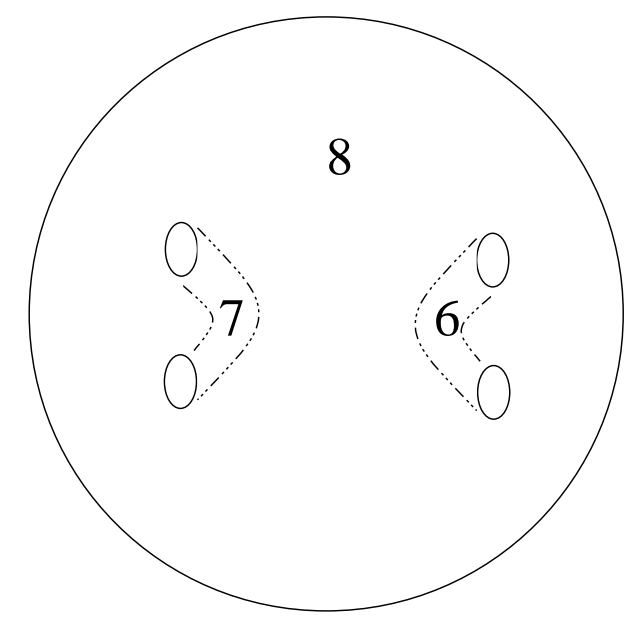

Fig. 6: The previous picture turned inside-out. The real slice is now the inside of the ball minus the two tunnels. The boundary of the ball is where $z_{8}=0$.

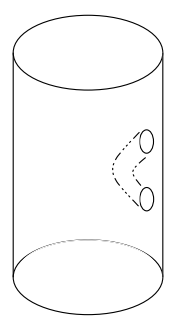

a

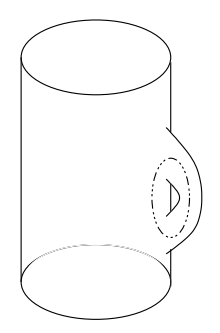

b
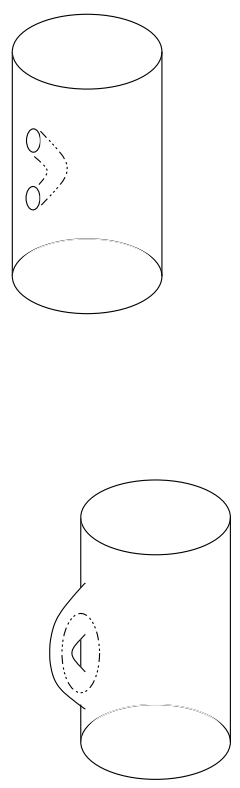

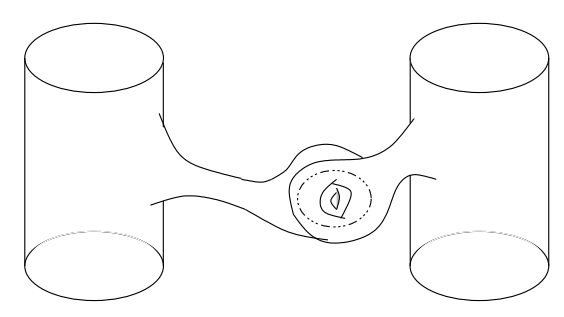

C

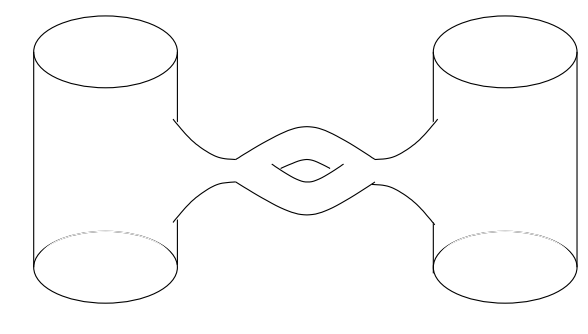

d

Fig. 7: Gluing handlebodies (in particular, solid cylinders) along a tunnel is the same as connecting them via a tube with a handle in it.

After we do this gluing, we find a solid genus 5 surface for each branch of $z_{8}$. The boundary of this surface is where $z_{8}=0$.

Since the two different branches for $z_{8}$ meet at $z_{8}=0$, we now glue two copies of the genus 5 surface together along their boundaries. In general, two solid genus $g$ surfaces glued in this manner describe a Heegaard splitting of a connected sum of $g$ copies of $S^{2} \times S^{1}$. Hence, this three-cycle has $b_{1}(\Sigma)=5$. 


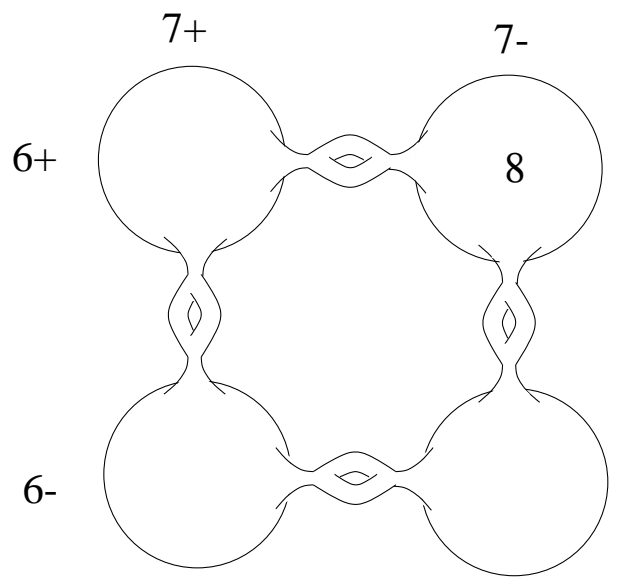

Fig. 8: Our special Lagrangian three-cycle is obtained by gluing two of these along their boundaries via the trivial identification. The numbers along the top and left indicate which branch each ball represents. 


\section{References}

[1] J.A. Harvey and G. Moore, "Superpotentials and membrane instantons," hepth/9907026.

[2] M.R. Douglas and M. Li, "D-brane realization of $\mathcal{N}=2$ super Yang-Mills theory in four dimensions," hep-th/9604041; A. Sen, "F-theory and orientifolds," Nucl. Phys. B475 (1996) 562, hep-th/9605150; T. Banks, M.R. Douglas and N. Seiberg, "Probing F-theory with branes," Phys. Lett. B387 (1996) 278, hep-th/9605199; N. Seiberg, "IR dynamics on branes and space-time geometry," Phys. Lett. B384 (1996) 81, hepth/9606017.

[3] M. R. Douglas, "Topics in D Geometry," Class. Quant. Grav. 17 (2000) 1057, hepth/9910170.

[4] I. Brunner, M.R. Douglas, A. Lawrence and C. Römelsberger, "D-branes on the Quintic," hep-th/9906200.

[5] P. Kaste, W. Lerche and C. Lutken, "D-branes on K3 Fibrations," hep-th/9912147.

[6] S. Kachru, S. Katz, A. Lawrence and J. McGreevy, "Open String Instantons and Superpotentials," hep-th/9912151.

[7] E. Scheidegger, "D-branes on Some One Parameter and Two Parameter Calabi-Yau Hypersurfaces," JHEP 0004 (2000) 003, hep-th/9912188.

[8] M. Naka, M. Nozaki, "Boundary states in Gepner models," JHEP 0005 (2000) 027, hep-th/0001037.

[9] B. Greene and C. Lazaroiu, "Collapsing D-branes in Calabi-Yau Moduli Space I," hep-th/0001025.

[10] I. Brunner and V. Schomerus, "D-branes at Singular Curves of Calabi-Yau Compactifications," JHEP 0004 (2000) 020, hep-th/0001132.

[11] C. Lazaroiu, "Collapsing D-branes in One Parameter Models and Small/Large Radius Duality," hep-th/0002004.

[12] M. Douglas, B. Fiol and C. Romelsberger, "Stability and BPS Branes," hepth/0002037.

[13] S. Govindarajan and T. Jayaraman, "On the Landau-Ginzburg Description of Boundary CFTs and Special Lagrangian Submanifolds," hep-th/0003242.

[14] M. Douglas, B. Fiol and C. Romelsberger, "The Spectrum of BPS Branes on a Noncompact Calabi-Yau Space," hep-th/0003263.

[15] F. Denef, "Supergravity Flows and D-brane Stability," hep-th/0005049.

[16] K. Hori, A. Iqbal and C. Vafa, "D-branes and Mirror Symmetry," hep-th/0005247.

[17] E. Witten, "Chern-Simons gauge theory as a string theory," in The Floer Memorial Volume, H. Hofer et. al., eds., Birkhauser (1995), Boston, hep-th/9207094.

[18] C. Vafa, "Extending mirror conjecture to Calabi-Yau with bundles," hep-th/9804131.

[19] H. Ooguri and C. Vafa, "Knot invariants and topological strings," hep-th/9912123. 
[20] H. Ooguri, Y. Oz and Z. Yin, "D-branes on Calabi-Yau spaces and their mirrors," Nucl. Phys. B477 (1996) 407, hep-th/9606112.

[21] E. Witten, "Mirror manifolds and topological field theory," in Mirror Symmetry I, S.-T. Yau (ed.), American Mathematical Society (1998), hep-th/9112056.

[22] J.A. Harvey and G. Moore, "On the algebras of BPS states," Comm. Math. Phys. 197 (1998) 489, hep-th/9609017.

[23] M. Marino, R. Minasian, G. Moore and A. Strominger, "Nonlinear instantons from supersymmetric p-branes," JHEP 0001 (2000) 005, hep-th/9911206.

[24] S. Kachru and J. McGreevy, "Supersymmetric three-cycles and supersymmetry breaking," Phys. Rev. D61 (2000) 026001, hep-th/9908135.

[25] K. Kodaira, "A Theorem of Completeness of Characteristic Systems for Analytic Families of Compact Submanifolds of Compact Manifolds," Ann. Math. 75 (1962) 146.

[26] P. Candelas, X. de la Ossa, A. Font, S. Katz and D.R. Morrison, "Mirror symmetry for two-parameter models - I," Nucl. Phys. B416 (1994) 481, hep-th/9308083.

[27] S. Katz, D.R. Morrison and M.R. Plesser, "Enhanced gauge symmetry in type II string theory," Nucl. Phys. B477 (1996) 105, hep-th/9601108.

[28] R. McLean, "Deformations of Calibrated Submanifolds", Duke Univ. PhD thesis, Duke preprint 96-01: see www.math.duke.edu/preprints/1996.html.

[29] B. Greene and M. Plesser, "Duality in Calabi-Yau Moduli Space," Nucl. Phys. B338 (1990) 15.

[30] E. Witten, "Phases of $\mathrm{N}=2$ theories in two dimensions," Nucl. Phys. B403 (1993) 159, hep-th/9301042.

[31] S.-T. Yau, "Calabi's conjecture and some new results in algebraic geometry," Proc. Nat. Acad. Sci. U.S.A. 74 (1977) 1798.

[32] A. Strominger, S.-T. Yau and E. Zaslow, "Mirror Symmetry is T-Duality," Nucl. Phys. B479 (1996) 243, hep-th/9606040.

[33] P.S. Aspinwall, B.R. Greene and D.R. Morrison, "The monomial-divisor mirror map," Internat. Math. Res. Notices 93 (1993) 319, alg-geom/9309007.

[34] M. Kontsevich, "Homological algebra of mirror symmetry," Proc. of the 1994 International Congress of Mathematicians, Birkhäuser (Boston) 1995, alg-geom/9411018.

[35] T. Banks, L.J. Dixon, D. Friedan and E. Martinec, "Phenomenology and conformal field theory: or, can string theory predict the weak mixing angle?," Nucl. Phys. B299 (1988) 613. 